\title{
The Reactivity of the Donor-Acceptor Complex of Titanium Tetrachloride and N-Methylformanilide in Solvothermal Processes of Titanium Dioxide Formation
}

\author{
A. M. NEMERYUK, M.M. LYLINA and PINCHUK YURIY ANATOLYEVICH \\ Federal State Unitary Enterprise "State Research Institute of Chemical Reagents and Pure Chemical \\ Substances"(FSUE “IREA”), 107076, Moscow, Bogorodskiy Val, 3, Russia. \\ ${ }^{*}$ Corresponding author: amnamn@mail.ru \\ http://dx.doi.org/10.13005/ojc/330233
}

(Received: December 30, 2016; Accepted: March 21, 2017)

\begin{abstract}
Represented work was done to identify possible reaction ways of titanium carboxamide complexes with various organic compounds, mainly alcohols, resulting in the formation of particles of titanium dioxide in non-aqueous media. The titanium tetrachloride and $\mathrm{N}$-methylformanilide complex was selected as the subject of research. We studied the reaction of this compound with various alcohols.
\end{abstract}

Keywords: Titanium dioxide, N-metiformanilid, Nucleophilic Addition to the azomethine, Epichlorohydrin, Formylation, Chlorination, Dehydration, Polyols.

\section{INTRODUCTION}

Titanium dioxide and various oxide systems containing titanium dioxide attract interest due to the presence of a catalytic, as well assignified photocatalytic activity. The possibilities of obtaining titanium dioxide and oxide systems in the form of nanoparticles having different geometrical shape were studied and nanorods, nanotubes, micro and mesoporous materials, etc. were obtained in such a way. Generally, hydrothermal or solvothermal syntheses are used to obtain such objects. During such syntheses, one or more reagents are often involved in a supercritical state, necessitating the use of special equipment to carry out the synthesis at over pressure.

The solvothermal methods, based on the reactions in non-aqueous media, have a number of advantages compared with hydrothermal methods, so it becomes possible to obtain non-hydrated oxides, oxide systems derived from reagents that are incompatible with aqueous media. Also solvothermal processes allow to finer control the morphological characteristics of nano-objects obtained, tosynthesize multilayer structures with organic templates, further removed during the thermal oxidative treatment. A common disadvantage of hydro and solvothermal 
methods for producing nanoscale objects based on titanium dioxide and oxide-related systems is the need for equipment adapted to operate at over pressure that limits the scalability of the process and leads to significant complication of production processes.

It is important to explore the possibility of carrying out synthetic procedures to obtain nanoobjects based on titanium dioxide without equipment designed to operate under over pressure while maintaining the positive characteristics associated with conventional solvothermal technologies.

Generally, the titanium sources in the preparation of titanium dioxide in non-aqueous media are titanium alkoxides and halides. Despite the fact that the titanium is capable of forming compounds, in which it has a valence from 1 to 4 , the area that includes different reactions of titanium compounds where its valence is 4 is the most extensively studied area. There are several examples of preparation of low-valent titanium derivatives by solvothermal methods. Thus, for example, the lower titanium nitrides were obtained by reacting tetrakis(dimethylamine) titanium with hydrazine derivatives. Similarly, the corresponding nitrides were obtained by reacting alkali metal amides in hydrocarbon medium with halides or amides of other transition metals such as tantalum, zirconium and hafnium ${ }^{1}$.

Thus, titanium IV compounds that are compatible with organic media can be considered as potential precursors of titanium dioxide.

On the other hand, the presence of compounds capable of serving as an oxygen source in the reaction medium is required for the formation of titanium dioxide. These can be alcohols, ethers, esters, carboxylic acids, ketones, amides and other compounds ${ }^{2-4}$.

Reactions resulting in the preparation of titanium dioxide in non-aqueous media can be divided into several categories:

1. Reaction of titanium halides with alcohols

2. Reaction of titanium alkoxides, carboxylates and acetylacetonates with alcohols.
3. Reaction of titanium alkoxides with ketones.

4. Reaction of titanium acetylacetonates with amines.

5. Thermal decay of titanium alkoxides in media allowing to maintain required temperature at atmospheric pressure of about $300^{\circ} \mathrm{C}$.

Each of these syntheses has its own specific features. For example, the reaction of titanium tetrachloride with alcohols is extremely violent reaction accompanied by release of organic by-products in large quantities; some of them are highly polymeric, resinous compounds which are difficult to remove in order to obtain the oxide particles. Kinetic features of these reactions make it difficult to control the shape and the size of the objects.

2-4 methods can be carried out only under conditions of the classical solvothermal synthesis, which is associated with low boiling temperatures of organic compounds that are the oxygen donors in these processes.

Thermal decay of alkoxidescan be carried out at atmospheric pressure in medium of high boiling solvents only if used alkoxides have a boiling temperatureat atmospheric pressure of more than $300^{\circ} \mathrm{C}$.

Certainly, the selection of such reagents for solvothermal synthesis of titanium dioxide in the form of nanoscale particles is of our main interest, which would allow carrying out the process at moderate temperatures and atmospheric pressure.

Among other potentially useful compounds, it is necessary to highlight the donor-acceptor complexes of titanium tetrachloride and formic acid amides. These compounds have a range of properties that allow to consider them as very promising titanium precursors in solvothermal processes for the production of nano-objects based on titanium dioxide in the synthesis under atmospheric pressure.

It is known that titanium tetrachloride is a strong Lewis acid capable of forming stable complexes with electron-excess compounds including organic acid amides. Compounds formed from acid amides having partial substitution, are 
usually polymeric compounds insoluble in common solvents, the composition of such complexes is unstable and changes over time. On the other hand, disubstituted carboxamides form stable monomeric complexes, which are composed of one molecule of titanium tetrachloride with two molecules of carboxamide. This is associated with the coordination number 6 that is typical for titanium IV. Up to the present moment, there was no sufficiently detailed understanding of the structure of these complexes and their reactivity. Represented work was done to identify possible reaction ways of titanium carboxamide complexes with various organic compounds, mainly alcohols, resulting in the formation of particles of titanium dioxide in non-aqueous media. The titanium tetrachloride and $\mathrm{N}$-methylformanilide complex was selected as the subject of research. We studied the reaction of this compound with various alcohols.

\section{EXPERIMENTAL}

Titanium tetrachloride and $\mathrm{N}$-methyl formanilide complex was obtained by reacting of the reagents in chlorinated hydrocarbon medium.

A solution of $18.9 \mathrm{~g}$ of titanium tetrachloride $(0.1 \mathrm{~mol})$ in $40 \mathrm{~g}$. carbon tetrachloride was added dropwise to a solution of $32.7 \mathrm{~g}(0.25 \mathrm{~mol}) \mathrm{N}$-methyl formanilide (MFA) in $70 \mathrm{~g}$. carbon tetrachloride with vigorous stirring. The exothermic reaction occurred and the reaction mass was heated to $55-60^{\circ} \mathrm{C}$ and stirred at this temperature until the end of the formation of fine precipitate, painted in bright yellow color. The reaction mixture was cooled in a water bath in order to control the temperature. The precipitate was filtered off, washed with carbon tetrachloride and held in a vacuum of water jet pump for 4 hours to constant weight. It was obtained $43 \mathrm{~g}$ of a yellow crystalline substance.

The titanium content was $10.36 \%$ (estimated 10.41). Yield was $94 \%$, melting point $122-124^{\circ} \mathrm{C}$ with decomposition.

Trichloromethane, dichloromethane can be used instead of carbon tetrachloride, but it is necessary to consider a higher solubility of complex in these solvents at room temperature and to cool the solutions before precipitation by filtration for a more complete product release.

A solution of $18.9 \mathrm{~g}$ of titanium tetrachloride $(0.1 \mathrm{~mol})$ in $50 \mathrm{~g}$ of dichloromethane was added dropwise to a solution of $32.7 \mathrm{~g}$ $(0.25 \mathrm{~mol}) \mathrm{N}$-methylformanilide (MFA) in $100 \mathrm{~g}$ of dichloromethane with vigorous stirring. The exothermic reaction was accompanied by boiling of solvent. The reaction mixture was stirred until the end of the formation of fine precipitate, painted in bright yellow color. The reaction mixture was cooled in an ice water bath in order to control the temperature. When the reaction mass reached the temperature of $01^{\circ} \mathrm{C}$ the precipitate was filtered off, washed with cooled dichloromethane and heldin a vacuum of water jet pump for 2 hours to constant weight. It was obtained $41 \mathrm{~g}$ of a yellow crystalline substance. Titanium content was $10.39 \%$ (estimated. 10.41).

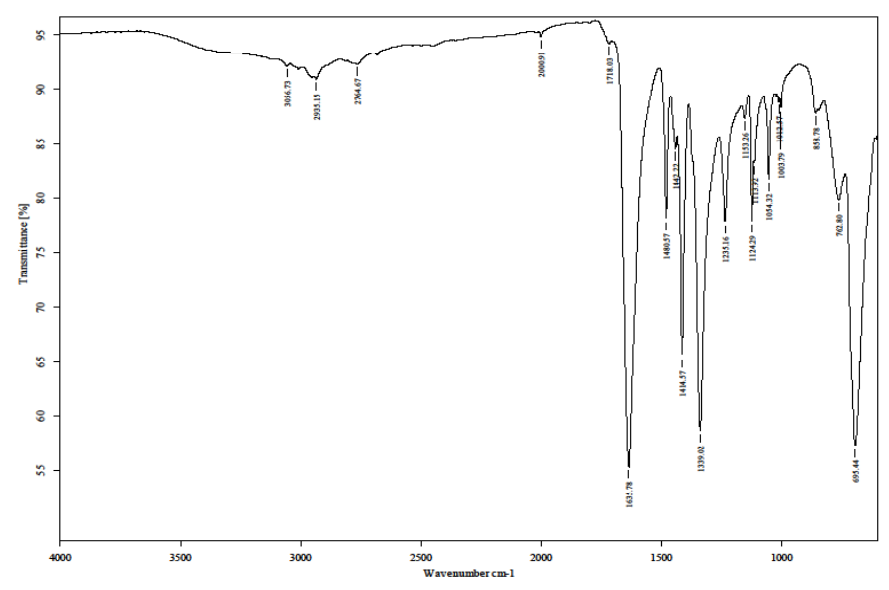

Fig. 1: IR spectrum of titanium tetrachloride and $\mathrm{N}$-methylformanilide complex 
Yield was $89.6 \%$, melting point $123-124^{\circ} \mathrm{C}$ with decomposition.

Figure 1 shows IR spectrum of the resulting complex, and Figure 2 shows IR spectrum of titanium tetrachloride and N,N-dimethyl formamide (DMFA) complex. This compound was prepared by the method described above ${ }^{5}$. As an example, below is the reaction product of IR spectrum of the titanium tetrachloride and DMFA complex with t-butanol, see Figure 3.

It is marked by the presence of the absorption band at $1633 \mathrm{~cm}^{-1}$ attributable to the carbonyl group of MFA, shifted toward lower values in comparison with the inherent amide carbonyl groups, not included in the complex sphere $\left(1730-1650 \mathrm{~cm}^{-1}\right)$, evidencing the participation of the carbonyl group of MFA in the formation of the complex compound. Similarly, DMFA-based compound is marked by a shift of carbonyl absorption band $\left(1632 \mathrm{~cm}^{-1}\right)$.

The reaction of MFA and titanium tetrachloride complex was studied using benzyl, cetyl alcohol, cyclohexanol and glycerol.

In order to compare the reactivity of the titanium alkoxidesimilar in the structure, they were obtained by interetherification method based on titanium tetraisopropoxide using the present method.
A mixture of titanium tetraisopropoxide and excess alcohol was heated under vigorous stirring in a round bottom flask equipped with magnetic stirrer, thermometer, inert gas inlet system and a descending condenser. If the reagent, for example, cetyl alcohol is a solid substance used at room temperature, then it should be pre-heated to a melting temperature that allows stirringthe mixture and eliminates local concentrated unevenness. It is observed the release of isopropanol vapors at a temperature of $80-90^{\circ}$ $C$ that are condensed and collected in a receiver equipped with a calcium chloride tube. Throughout the entire process, the current of dry argon is passed through the reaction device.

After separation of the theoretical amount of isopropanol, the reaction is considered completed. The mixture is cooled to room temperature under argon currentorthe heating is continued in order to study the thermal decomposition products of the higher titanium alkoxides formed. It should be noted that as a result of the reaction of titanium tetraisopropoxide with glycerol it is formed a white solid compound that decomposes without melting with acrolein emission. Hypothetically, this compound is a space polymer. Thus, it is necessary to use $20-40$ fold excess of the polyol when using the glycerol to provide the reaction mixture with viscosity sufficient for mixing.

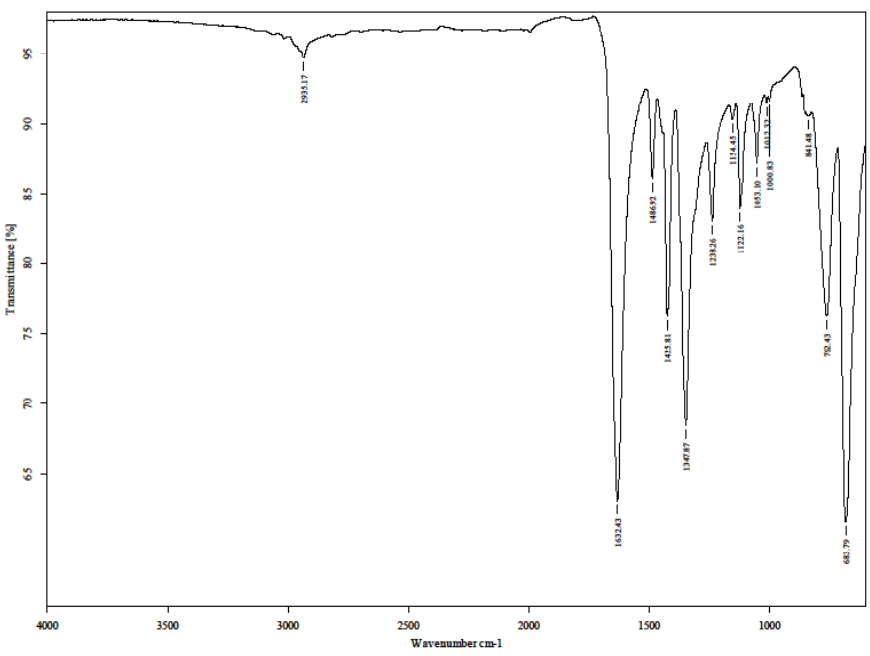

Fig. 2: IR spectrum of titanium tetrachloride and $\mathrm{N}, \mathrm{N}$-dimethylformamide complex 
Thermal decomposition of titanium alkoxides is observed at temperatures $290-300^{\circ} \mathrm{C}$. Cetyl alcohol was recognized as the bestalkoxide decomposition mediumaccording to the range of its characteristics, having a high boiling point at atmospheric pressure and being inert compound to enable the occurrence of side reactions leading to the formation of resinous products.

Reaction with alcohols proceeds stepwise, when using MFA and titanium tetrachloride complex.
Depending on the reactivity of alcohol, various products of dehydration and chlorination can be obtained. Thus, the reaction of glycerol with MFA and titanium tetrachloride complex furnishes epichlorohydrin together with resinous products. Cyclohexanol furnishes cyclohexene, benzyl alcohol furnishes a mixture of products among which the main component is benzyl chloride, but mixture also containsbenzyl formate, toluene, dibenzyl ether, and other compounds.

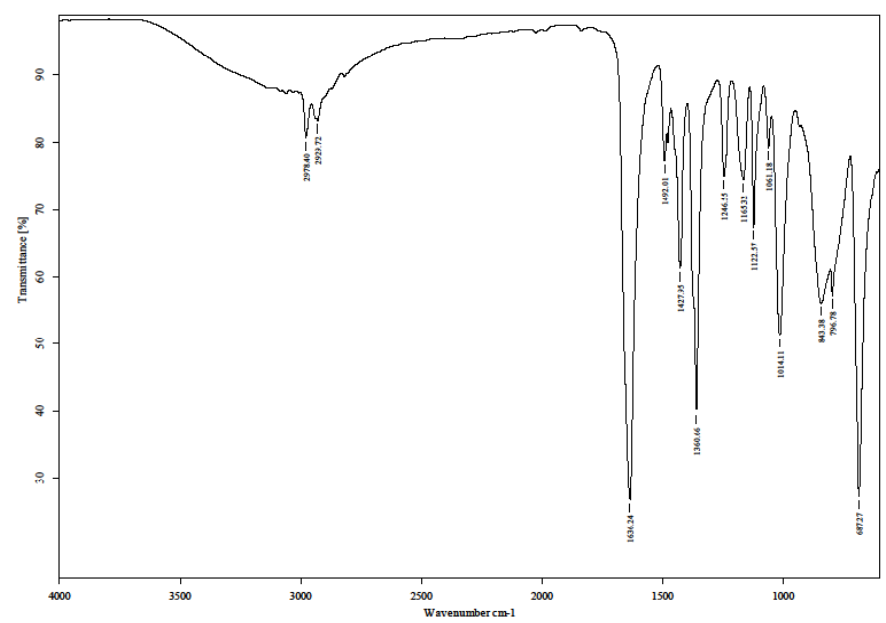

Fig. 3: IR spectrum of the adduct of titanium tetrachloride and DMFA complex with t-butanol
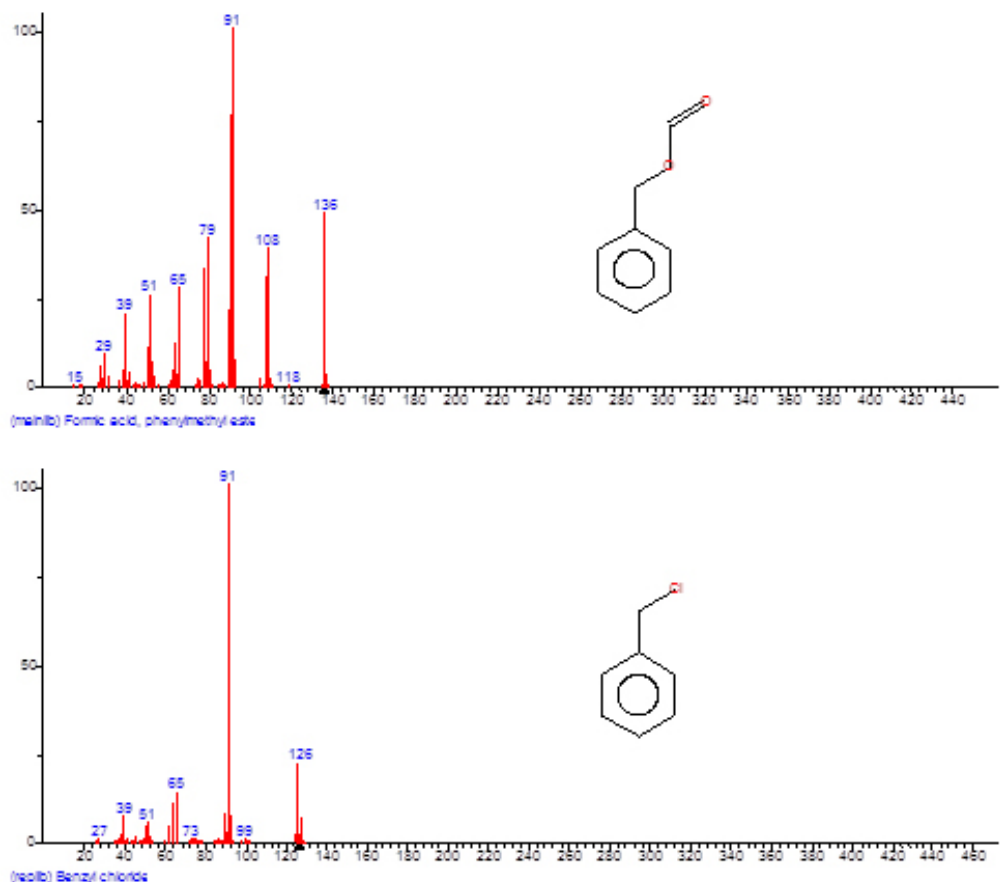
(vegib) Bsnz/ criconds 

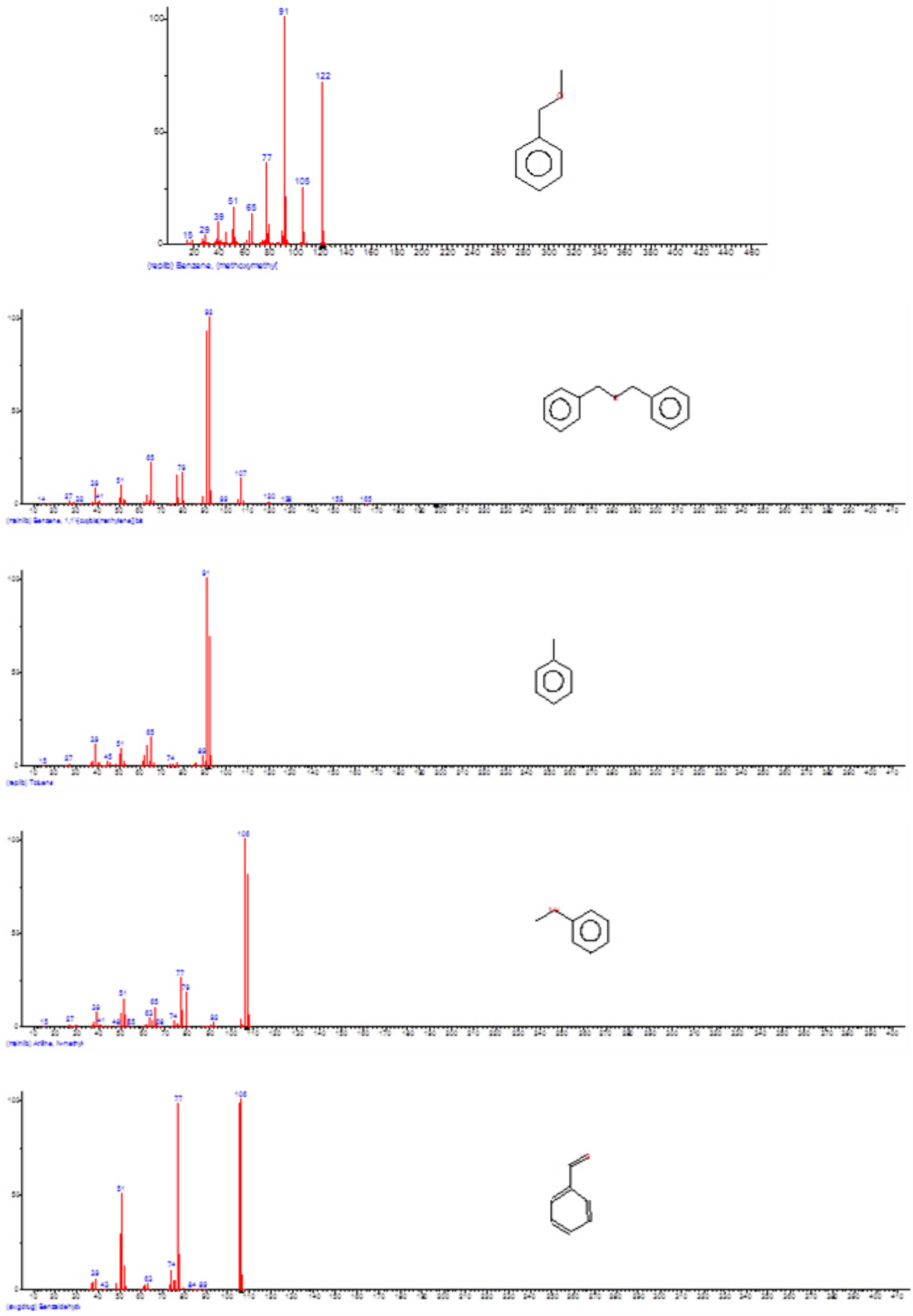
Particular interest is associated with the detection of the triazene derivatives among the reaction products of MFA and titanium tetrachloride complex:

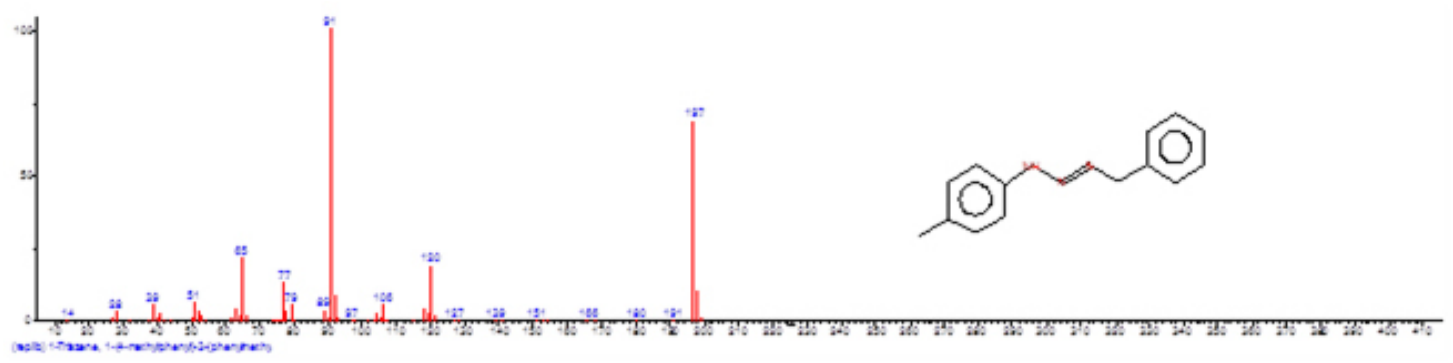

Discovered products show the complexity of the course of a reaction of the complex with alcohols, accompanied by oxidation - reduction processes, as evidenced by the presence of toluene and benzaldehyde in the composition of the reaction products. The specified structure of triazene can be formed after the occurrence of $p$-toluidine in a reaction mixturethat was formed fromMFA deformylating product- $\mathrm{N}$ - methylanilineor imonium compounds.

Thus, it follows from received data that alcohol adduct was originally formed to activated product by complexation of MFA with titanium tetrachloride. At this stage the initially bright yellow complex solution is decolorized, but the release of hydrogen chloride is not observed. The process of thermal decomposition of the resulting compound flows with further increase in temperature, accompanied by release of a complex mixture of products. Accordingly, the primary act of alcohols reaction with titanium tetrachloride and MFA complex is addition to the activated double bond, as shown in Scheme 1.

The resulting product under heat loses hydrogen chloride, which is actually observed, Scheme 2
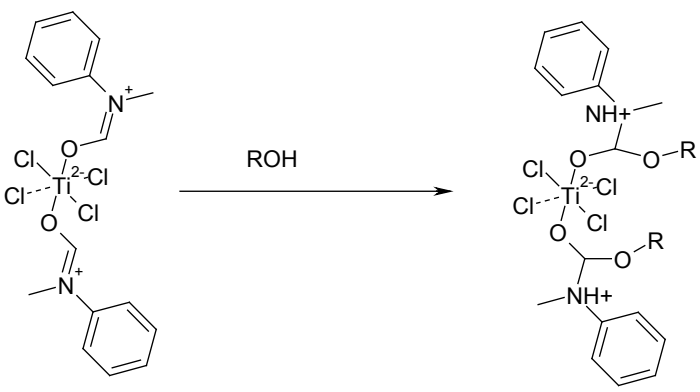

Scheme 1
With further increase in temperature it is observed a decay of resulting compound with release of titanium dioxide, hydrogen chloride or chlorine containing products and numerous organic compounds, mainly, unsaturated compounds or formates, formed on the basis of alcohols used. In this case, if the used alcohol easily replaces the hydroxyl group with chlorine, as ithappens when using benzyl alcohol, then corresponding halogen derivative such as benzyl is found among the reaction products. Cyclohexanol or higher aliphatic alcohols furnish the unsaturated compounds. Of particular interest is the reaction process of glycerin with titanium tetrachloride and MFA complex. Thus, epichlorohydrin is formed as a primary reaction product together with other compounds. In this case, dehydrating and chlorinating ability of the complex is clearly manifested.

It should be noted that the temperature of decomposition of such compounds is usually substantially lower than that of tetra-replaced titanium alkoxides. In the latter case it reaches 290-310 ${ }^{\circ} \mathrm{C}^{6-12}$, whereas the reaction products of alcohols with titanium tetrachloride and MFA complex decompose with release of titanium dioxide at temperatures $180-220^{\circ} \mathrm{C}$.
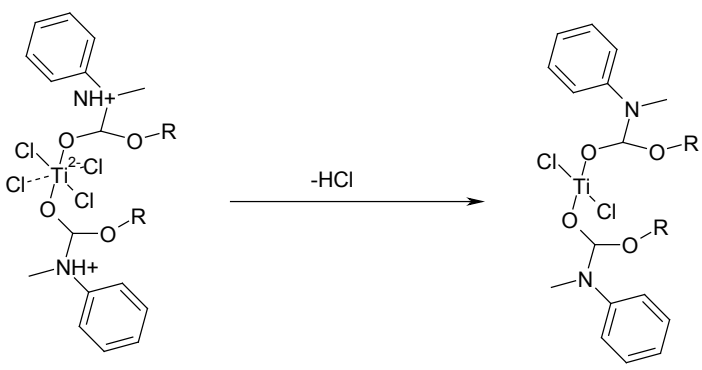

Scheme 2 
Moreover, this process is preceded by polymerization as evidenced by the significant increase in viscosity of the reaction mass within the temperature range of $150-180^{\circ} \mathrm{C}$. Currently, there is no certainty quite how the polymer chain isbuilt in such compounds, whether Ti-O-Ti fragments or Ti-O- $(\mathrm{CH}(\mathrm{N}(\mathrm{CH} 3) \mathrm{Ph}))$ - O-Ti are its elementary components or both options simultaneously. Indirect evidence in favor of the option assuming the presence of anorganic nitrogen-containing bridge between the titanium atoms consists in the lack of proper thermal decomposition of MFA in the products. It is most likely that the decomposition occurs in several directions at the same time and a complex mixture of polymers decomposes, which explains the variety of reaction products.

\section{CONCLUSION}

Based on the study of the reaction of titanium tetrachloride complex with MFA, it was found that during the reaction with alcohols, the addition occurred by activated $\mathrm{C}=\mathrm{N}^{+}$linkage that was accompanied by formation of unstained compounds, unlike the original complex. Titanium dioxide and various organic products of dehydration, formylation and halogenation of initial alcohols were formed during thermal degradation of the resulting products. Dehydration can resultin formation of ethers, including cyclic, and can result in formation of unsaturated compounds.

The organic resinous product formation is minimized when using the higher aliphatic alcohols. Thus, the use of titanium tetrachloride and MFA complex during the process of solvothermal synthesis oftitanium dioxide in higher alcohol medium allows carrying out this process at atmospheric pressure and at temperatures ranging $180-200^{\circ} \mathrm{C}$. This offers the prospect of using this compound for producing hybrid organo-mineral composites based on organic polymers exposed to thermal degradation at elevated temperatures, it becomes possible to use alcohols adducts with titanium tetrachloride and MFA complex as a precursor of titanium dioxide when creating a variety of thin-film structures obtainedby solution-type methods or to obtain nano-dispersed oxide systems based on titanium dioxide.

\section{ACKNOWLEDGMENT}

Applied researches are carried out with financial support of the state by the Russia Ministry of Education and Science under Grant Agreement No.14.624.21.0006 of September 8, 2014 (Unique identifier for Applied Scientific Researches (project) RFMEFI62414X0006).

\section{REFERENCES}

1. Mazumder B., Chirico P. and Hector A. L. Inorg. Chem., 2008, 47(20), 9684-9690

2. Niederberger M., Garnweitner G.Chemistry,2006,12(28), 7282-302

3. Pinna N. and Niederberger M. Angew. Chem. Int. Ed. 2008, 47(29), 5292 - 5304

4. Tätte T., Hussainov M., Paalo M., Part M., Talviste R., Kiisk V., Mändar H., Põhako K., Pehk T., Reivelt K., Natali M., Gurauskis J., Lõhmus A., Mäeorg U.SciTechnolAdv Mater. 2011, 12(3), 034412

5. Arehambault J., Rivest R. Canadian J. of Chemistry, 1958, 36

6. Choi H.-J., Kim J.-S., Kang M. Bull. Korean Chem. Soc. 2007, 28, 581
7. Li H.,Bian Z., Zhu J.,Huo Y., Li H., LuY. J. Am. Chem. Soc.2007, 129, 4538

8. Valentin C. D.,Pacchioni, G.,Selloni A. Chem. Mater. 2005, 17,6656

9. Usseglio S.,DaminA.,Scarano D.,Bordiga S.,Zecchina A.,Lamberti C. J.Am. Chem. Soc. 2007, 129, 2822

10. Morandeira A., Lopez-Duarte I., Martinez-Diaz M. V.,O'Regan B., Shuttle C., Haji-Zainulabidin N. A., Torres T.,Palomares E.,Durrant J. R. J.Am. Chem. Soc. 2007, 129, 9250

11. Tang J.,Quan H., Ye J.Chem. Mater. 2007, 19, 116

12. Zan L., Liu Z.,Zhong J., Peng Z. J. Mater. Sci. 2004, 39, 3261 